\title{
Learning Based Non-rigid Multi-modal Image Registration Using Kullback-Leibler Divergence
}

\author{
Christoph Guetter ${ }^{1,2}$, Chenyang $\mathrm{Xu}^{1}$, Frank Sauer ${ }^{1}$, and Joachim Hornegger ${ }^{2}$ \\ 1 Imaging \& Visualization Department, Siemens Corporate Research, Princeton, USA \\ \{christoph.guetter, chenyang.xu, frank.sauer\}@siemens.com \\ 2 Institute of Computer Science, Universität Erlangen-Nürnberg, Erlangen, Germany \\ joachim.hornegger@informatik. uni-erlangen.de
}

\begin{abstract}
The need for non-rigid multi-modal registration is becoming increasingly common for many clinical applications. To date, however, existing proposed techniques remain as largely academic research effort with very few methods being validated for clinical product use. It has been suggested by Crum et al. 1] that the context-free nature of these methods is one of the main limitations and that moving towards context-specific methods by incorporating prior knowledge of the underlying registration problem is necessary to achieve registration results that are accurate and robust enough for clinical applications. In this paper, we propose a novel non-rigid multi-modal registration method using a variational formulation that incorporates a prior learned joint intensity distribution. The registration is achieved by simultaneously minimizing the Kullback-Leibler divergence between an observed and a learned joint intensity distribution and maximizing the mutual information between reference and alignment images. We have applied our proposed method on both synthetic and real images with encouraging results.
\end{abstract}

\section{Introduction}

Non-rigid multi-modal image registration in medical applications has become increasingly important to physicians in recent years. The fusion of complimentary image information has been shown to be particularly beneficial to physician's diagnosis. Furthermore, new imaging techniques such as molecular imaging pose a huge demand for multi-modal image registration in order to show functional, anatomical, and/or molecular image information in a single fused image.

Multi-modal image registration is a challenging problem. It has been strongly influenced by the introduction of an information theoretic similarity measure, the well-known mutual information (MI), into the medical registration domain in 1995 [2, 3]. Amongst others, MI has been applied successfully to rigid as well as non-rigid multi-modal registration of medical images. Surveys regarding this topic have also been published recently [4, 5]. Nevertheless, drawbacks of MI became apparent especially when the underlying transformation is not originated from a low dimensional parameter space, i.e. for non-parametric or non-rigid transformation models. Both the non-convexity of MI and an unconstrained transformation model make non-rigid multi-modal image registration a 
very challenging problem. Extensive research along this direction has been performed in recent years including variational formulations using advanced regularizers [6, 7], and local similarity maximization [6, 8, Most non-rigid multi-modal registration work proposed so far focuses on methods that do not consider the underlying context of the registration such as the intensity mapping relationship of the class of images to be registered, statistics of modalities to be registered, and other prior information about the registration problem. It has been suggested by Crum et al. [1] that the context-free nature of these non-rigid registration methods is one of the main limitations for them to be clinically useful and that moving towards context-specific methods by incorporating prior knowledge of the underlying registration problem is necessary to achieve accurate and robust registration results.

In the case of rigid multi-modal image registration several approaches have been proposed to use prior information during optimization [9, 10, 11. Leventon and Grimson were the first to use a prior learned joint intensity distribution 9, where the registration is obtained by maximizing the log likelihood of the images to be registered. Zöllei et al. showed that this method makes some implicit assumptions about the desired solution which do not always hold 12 . Chung et al. found empirically that the minimization of the Kullback-Leibler (KL) divergence between an observed and a learned joint intensity distribution is superior to maximizing the log likelihood [10, 11. For non-rigid multi-modal image registration, however, very few work has been published so far. In [6], Hermosillo et al. proposed a supervised non-rigid registration algorithm using ML. Although KL divergence has been used in context-free non-rigid registration work [13], context-specific non-rigid multi-modal registration using KL divergence, to the best of our knowlege, has not been reported to date.

In this paper, we propose a variational formulation that incorporates prior knowledge by minimizing the KL divergence between an observed and a learned joint intensity distribution. In addition to the KL divergence term, our formulation also incorporates a regularization term that regularizes the displacement field and a term that maximizes the MI between reference and alignment images. Our work can be seen as an extension to the variational formulation work by Hermosillo et al. 6].

The outline of the paper is as follows. Section 2 describes the proposed method, the derivatives used for the optimization process, and its implementation. In Section 3, experiments on both synthetic and real images are presented to validate the proposed method. We conclude in Section 4 with a discussion and future developments.

\section{Description of Method}

\subsection{Registration by Driving Mutual Information with Prior Knowledge}

In order to non-rigidly match images from two different modalities, several strategies have been proposed in the past. Generally speaking, there are two categories 
of solutions available. The first approach, studied extensively in recent years, considers maximizing one or multiple similarity measures defined on both reference and alignment images such as intensity, gradient, edges, landmarks, shapes, and so on. The second approach uses prior knowledge obtained from pre-registered trained data to get a solution that is more meaningful in the clinical context. Our proposed method combines both perspectives into a unified formulation by simultaneously encouraging the observed joint intensity distribution to resemble the expected joint intensity distribution learned a priori and maximizing a similarity measure. This can be intuitively understood as guiding a context-free similarity measure by prior knowledge.

We define our combined registration framework as the minimization of the following cost functional

$$
\begin{aligned}
\mathcal{J}(\mathbf{u}) & =\alpha \mathcal{I}_{\mathbf{M I}}(\mathbf{u})+(1-\alpha) \mathcal{I}_{\mathbf{K L}}(\mathbf{u})+\lambda \mathcal{R}(\mathbf{u}), \quad \alpha \in[0,1], \lambda \in \mathbb{R}_{+} \\
\hat{\mathbf{u}} & =\operatorname{argmin} \mathcal{J}(\mathbf{u}),
\end{aligned}
$$

where $\mathbf{u}$ is a displacement field, $\mathcal{R}$ defines regularization or smoothing on $\mathbf{u}$, and $\lambda$ is a positive constant that decides the amount of regularization. $\mathcal{I}_{\mathbf{K L}}$ measures the KL divergence between observed and learned data, and $\mathcal{I}_{\text {MI }}$ denotes an expression for MI of the observed data. Here, we realize the role that prior knowledge plays. A displacement field that maximizes MI is being steered by prior information to achieve accurate alignment. The factor $\alpha$ controls the amount of guidance through prior knowledge. For $\alpha=0$ the registration problem is solely based on the prior information. For $\alpha=1$ the registration is defined as the classical optimization of MI without any prior information. For $\alpha \in(0,1)$, the maximization of MI is driven by clinical context in the form of prior knowledge captured by the minimization of the KL divergence.

This prior knowledge can be acquired in several ways and has become more accessible recently. One can use the expert knowledge of a physician who manually aligns the images or one can leverage the fused imaging data acquired using the dual-modality (PET/CT, SPECT/CT), also known as hybrid, scanners. The latter provide extensive amounts of pre-registered data, which is very important for avoiding patient specific training data. In order to increase robustness, one may learn a joint density distribution that represents a mean prior information of $n$ pre-aligned images. But it has to be examined carefully as most scanners cannot correct the misalignment due to organ movement.

\subsection{Derivative of Kullback-Leibler Divergence and Mutual Information}

In the following we will refer to the two images that are to be registered by the functions $f_{1}: \Omega \subset \mathbb{R}^{n} \mapsto \mathbb{R}$ and $f_{2}: \Omega \subset \mathbb{R}^{n} \mapsto \mathbb{R}$. The images are registered by retrieving the underlying displacement field. Given the images, a displacement field can be modeled by a mapping $\mathbf{u}: \Omega \mapsto \Omega$. Without loss of generality, we can denote $f_{1}$ as the reference image and $f_{2}$ as the alignment image during the registration process. 
We indicate by $p_{1}^{o}\left(f_{1}\right), p_{2}^{o}\left(f_{2}\right)$ and $p_{\mathbf{u}}^{o}\left(f_{1}, f_{2}\right)$ the marginal and joint intensity distributions estimated from $f_{1}(\mathbf{x})$ and $f_{2}(\mathbf{x}+\mathbf{u}(\mathbf{x}))$ respectively. $p^{\ell}\left(f_{1}, f_{2}\right)$ is an estimate for the joint intensity distribution of the training data. In practice, the distributions are estimated by using a non-parametric Parzen window estimator with a Gaussian as the windowing function.

We incorporate prior knowledge by minimizing the KL divergence between observed and trained data. The KL divergence for a given displacement field $\mathbf{u}$ can be expressed as:

$$
\mathcal{I}_{\mathbf{K L}}(\mathbf{u})=\int_{\Omega} p_{\mathbf{u}}^{o}\left(i_{1}, i_{2}\right) \ln \frac{p_{\mathbf{u}}^{o}\left(i_{1}, i_{2}\right)}{p^{\ell}\left(i_{1}, i_{2}\right)} d \mathbf{x}
$$

where $i_{1}=f_{1}(\mathbf{x})$ and $i_{2}=f_{2}(\mathbf{x}+\mathbf{u}(\mathbf{x}))$. The MI-based objective function is defined as the negate MI between the reference image and the alignment image transformed by $\mathbf{u}$ and can be expressed as:

$$
\mathcal{I}_{\mathbf{M I}}(\mathbf{u})=-\int_{\Omega} p_{\mathbf{u}}^{o}\left(i_{1}, i_{2}\right) \ln \frac{p_{\mathbf{u}}^{o}\left(i_{1}, i_{2}\right)}{p_{1}^{o}\left(i_{1}\right) p_{2}^{o}\left(i_{2}\right)} d \mathbf{x},
$$

We notice that MI can be viewed as the KL divergence between the observed joint density and the product of the observed marginals, whereas in $\mathcal{I}_{\mathbf{K L}}$ the product of the marginal densities is replaced by the prior knowledge learned from training data. Note that we use the negate of the MI here to define a cost.

The minimum of (1) can be found by means of variational calculus. We may descend the gradient of the combined functional with respect to the displacement field. The gradient of (1) is defined as,

$$
\nabla_{\mathbf{u}} \mathcal{J}=\alpha \nabla_{\mathbf{u}} \mathcal{I}_{\mathbf{M I}}+(1-\alpha) \nabla_{\mathbf{u}} \mathcal{I}_{\mathbf{K L}}+\lambda \nabla_{\mathbf{u}} \mathcal{R}
$$

The gradient of MI has been derived by Hermosillo et al. in 6. To derive the gradient of the KL divergence, we use the definition for a non-parametric Parzen density model. After some manipulation, $\nabla_{\mathbf{u}} \mathcal{I}_{\mathbf{K L}}$ can be written as follows,

$$
\begin{aligned}
\nabla_{\mathbf{u}} \mathcal{I}_{\mathbf{K L}}= & -\frac{1}{\mathrm{~N}}\left[\left(\frac{\partial_{2} p_{\mathbf{u}}^{o}\left(i_{1}, i_{2}\right)}{p_{\mathbf{u}}^{o}\left(i_{1}, i_{2}\right)}-\frac{\partial_{2} p^{\ell}\left(i_{1}, i_{2}\right)}{p^{\ell}\left(i_{1}, i_{2}\right)}\right) * \mathrm{G}_{\sigma}\right]\left(f_{1}(\mathbf{x}), f_{2}(\mathbf{x}+\mathbf{u}(\mathbf{x}))\right. \\
& \cdot \nabla f_{2}(\mathbf{x}+\mathbf{u}(\mathbf{x})) .
\end{aligned}
$$

Here, $\mathbf{G}_{\sigma}$ is a two-dimensional Gaussian with standard deviation $\sigma, \partial_{2}$ is the partial derivative of a function with respect to its second variable, and $\mathrm{N}$ is a normalizing constant. We immediately notice the term $\frac{\partial_{2} p_{\mathbf{u}}^{o}\left(i_{1}, i_{2}\right)}{p_{\mathbf{u}}^{o}\left(i_{1}, i_{2}\right)}-\frac{\partial_{2} p^{\ell}\left(i_{1}, i_{2}\right)}{p^{\ell}\left(i_{1}, i_{2}\right)}$ as the comparison function of our registration method. This comparison function is evaluated repeatedly during the registration. In fact, alignment is achieved by continous adjustments of the joint intensity model until it resembles the learned joint intensity distribution. Furthermore, this assessment shows the central difference of our KL-based approach from the ML approach in [6], where the observed joint intensity distribution remains static. 


\subsection{Implementation}

Variational calculus allows us to compute the minimizing displacement field by descending along the gradient $\nabla_{\mathbf{u}} \mathcal{J}$. We get the classical gradient flow:

$$
\begin{aligned}
& \mathbf{u}_{t}=-\nabla_{\mathbf{u}} \mathcal{J} \\
& \mathbf{u}(\cdot, 0)=\mathbf{u}_{0}
\end{aligned}
$$

with $\mathbf{u}_{0}$ being a suitable initial guess for the displacement field. In this paper, we use a Tikhonov model for regularization, i.e. $\mathcal{R}[\mathbf{u}]=\frac{1}{2} \int_{\Omega}|\nabla \mathbf{u}(\mathbf{x})|^{2} d \mathbf{x}$. Its gradient expression is: $\nabla_{\mathbf{u}} \mathcal{R}[\mathbf{u}]=\operatorname{div}\left(\frac{\mathcal{R}^{\prime}[\mathbf{u}]}{|\nabla \mathbf{u}|} \nabla \mathbf{u}\right)=\operatorname{div}(\nabla \mathbf{u})=\Delta \mathbf{u}$, where $\Delta$ denotes the Laplace operator. Starting from an initial guess, we will follow a gradient descent strategy to find a solution for (1). In order to recover a larger class of deformations, to decrease computational cost, and to avoid irrelevant extrema of the non-convex functional, we pursue a coarse to fine scheme, i.e. consecutively smoothing and subsampling the images.

\section{Experiments}

Phantom Registration. The following phantom images were created to point out the importance of using context-specific information. Figure 1 visualizes an ambiguous setting for non-rigid registration. A circle is registered non-rigidly to another one that is of different intensity. However, its location is chosen such that there is an overlap with two other circles, a smaller and a larger circle, in a joint image. This setup suggests that there are at least two eqivalent optima for a context-free distance measure to align the circles. We compare two methods, i.e. minimizing (1) with $\alpha=1$ and $\alpha=0$ respectively. We train that the circle should align to the small circle. The MI method, $\alpha=1$, finds an optimum in registering to the big circle, Figs. $1(\mathrm{~d})$ and $1(\mathrm{e})$, whereas the KL approach, $\alpha=0$, registers to the small circle, Figs. $1(\mathrm{f})$ and $1(\mathrm{~g})$. Note that using the KL approach, we could also train the algorithm to align to the big circle.

T1 - T2 MRI Registration. We tested the KL method ( $\alpha=0)$ on a simulated T1/T2 MRI brain data set acquired from the Brain Web Simulated Brain

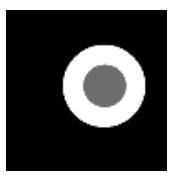

(a)

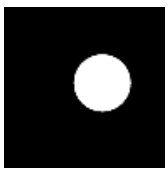

(b)

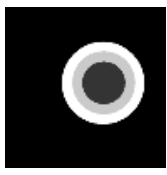

(c)

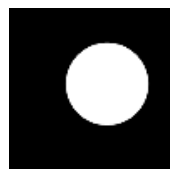

(d)

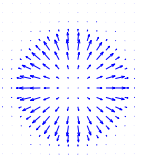

(e)

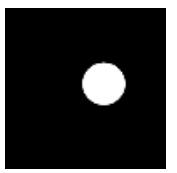

(f)

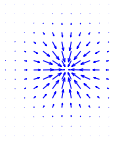

(g)

Fig. 1. (a) reference image $(512 \times 512)$, (b) alignment image, (c) difference image, (d) image (b) aligned using MI criterion only, (e) retrieved displacement field for (d), (f) image (b) aligned using prior information only, and (g) retrieved displacement field for(f) 


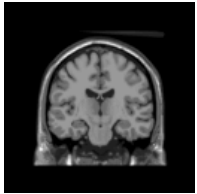

(a)

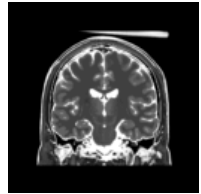

(b)

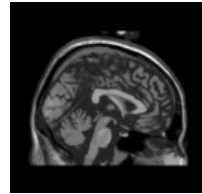

(c)

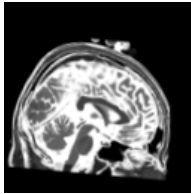

(d)

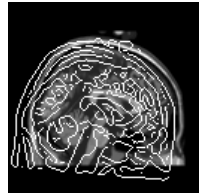

(e)

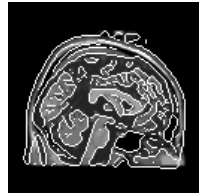

(f)

Fig. 2. (a), (b) Training slices for T1-T2, (c) reference image, (d) alignment image, (e) edge map of unregistered $\mathrm{T} 2$ slice superimposed on $\mathrm{T} 1$ slice, and (f) registered result

Database [14. The coronal slices, Fig. 2(a) and 2(b), were used for training whereas registration was performed on the sagittal slices, Fig. 2(c) and 2(d) The T2 image has been deformed by an artificially created displacement field. This experiment shows the strength of training joint intensity distributions that are used successfully for non-rigid registration.

SPECT - CT Registration. Our next experiment is performed on two corresponding slices of a SPECT/CT data set acquired by a Siemens Symbia T2 SPECT/CT hybrid scanner. We generate our prior knowledge from those two slices and deform the SPECT slice by an artificial displacement field. MI $(\alpha=1)$ and $\mathrm{KL}(\alpha=0)$ are compared for performance and the final registration results are visualized in Fig. 3. Since we have the ground truth, we computed the difference between the warped SPECT images and the original image for visual evaluation. From the difference images we notice that although we are using a multi-resolution strategy, the MI-based approach gets trapped in an irrelavant local minimum possibly due to its insensitivity to local deformation. This experiment demonstrates the potential benefit of incorporating prior knowledge for registration in clinical applications.

PET - CT Registration. Our last experiment describes a PET/CT registration from clinical practice involving a visual evaluation by an expert. The imaging data acquired from a 70 year old male patient with multiple lesions in the lung and was acquired by a Siemens Sensation 10 (CT) and a Siemens Ecat 926 (PET). The PET was acquired 6 days after the CT. According to the

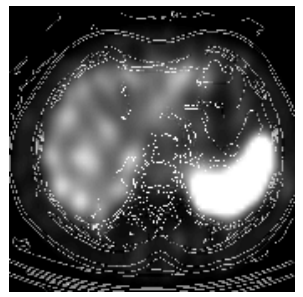

(a)

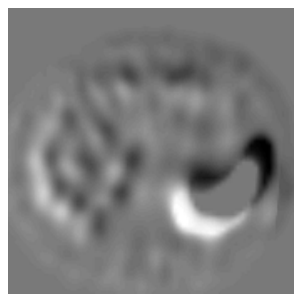

(b)

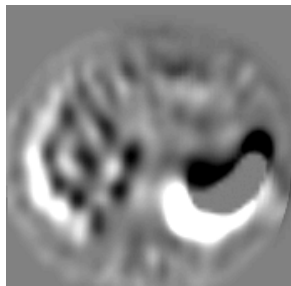

(c)

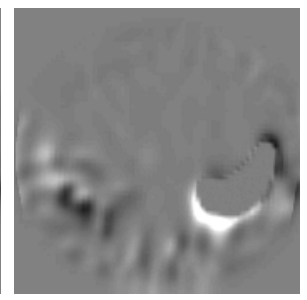

(d)

Fig. 3. (a) Edge map of CT slice overlayed on SPECT slice as acquired from scanner, (b) deformed SPECT difference image, (c) SPECT difference image for MI based approach, (d) SPECT difference image for KL based approach 


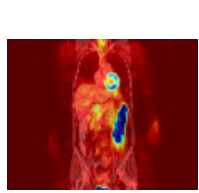

(a)

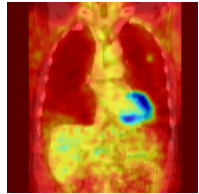

(b)

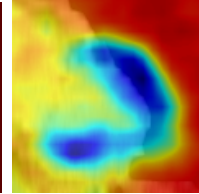

(c)

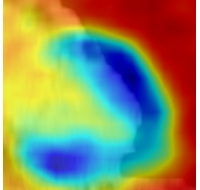

(d)

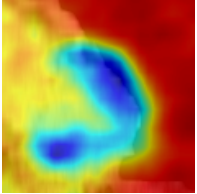

(e)

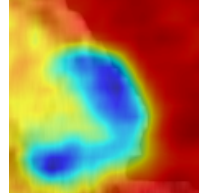

(f)

Fig. 4. Blended PET/CT images showing (a) training data, (b) misaligned slices, (c) misaligned slices (zoomed), (d) pure MI registration (zoomed), (e) pure KL registration (zoomed) (e) combined result (zoomed), $40 \% \mathrm{MI}$ and $60 \% \mathrm{KL}$

evaluation of an expert physician, only parts of the volume were registered accurately by a preceding manual fusion. For our experiment, we trained on two slices that have been classified as good registration and performed our approach on a misaligned slice. Figure 4 shows a collection of overlayed CT and corresponding PET images. In addition to a strong misalignment of the cardiac ventricle, an alignment deficit in the contours of the liver, the mediastinum and the thorax can be seen in Fig. 4(b). Figures 4(d) and 4(e) show the results for both the pure MI- $(\alpha=1)$ and the pure KL-based $(\alpha=0)$ registration, whereas Fig. 4(f) illustrates the registration result for our combined approach $(\alpha=0.4)$. The physician evaluated the combined approach as the most accurate one among all three registration results due to its accuracy not only in the alignment of the heart but also in the matching of the thoracic, mediastinal and hepatical (liver) outlines.

\section{Discussion and Conclusion}

We presented a novel approach to non-rigid multi-modal image registration by using prior information. The proposed framework allows flexible adjustment for the available quality of prior knowledge. Preliminary experiments on synthetically created phantoms and on real MRI, SPECT/CT, and PET/CT data show that prior knowledge can be crucial for retrieving the correct underlying displacement field. In addition, we have shown that our method has improved performance over a context-free registration. Future directions of research include the extension of our approach to $3 \mathrm{D}$, an adaptive selection of the steering parameter $\alpha$ across different levels of resolution, and an investigation of a more comprehensive training data representation, which goes beyond using the mean joint intensity distribution. In order to confirm the robustness and the accuracy of this approach for multi-modal datasets, a more complete qualitative and quantitative experimental study must be carried out.

Acknowledgements. The authors would like to thank Christophe Chefd'Hotel, Lilla Zöllei, Daniel Cremers, and Klaus Kirchberg for helpful discussions. We are grateful to Gabi Wolz for her expert clinical knowledge in validating our results. The authors gratefully acknowledge the financial support by Dr. Jim Williams at Siemens Corporate Research Inc. and the Deutsche Forschungsgesellschaft (DFG) through grant SFB 603, TP C10. 


\section{References}

[1] Crum, W., Griffin, L., Hill, D., Hawkes, D.: Zen and the art of medical image registration: correspondence, homology, and quality. NeuroImage 20 (2003) 14251437

[2] Wells, W., Viola, P., Atsumi, H., Nakajima, S., Kikinis, R.: Multi-modal volume registration by maximization of mutual information. MIA 1 (1996) 35-51

[3] Maes, F., Collignon, A., Vandermeulen, D., Marchal, G., Suetens, P.: Multimodality image registration by maximization of mutual information. IEEE TMI 16 (1997) 187-198

[4] Pluim, J.P.W., Maintz, J.B.A., Viergever, M.A.: Mutual-information-based registration of medical images: a survey. IEEE TMI 22 (2003) 986-1004

[5] Maes, F., Vandermeulen, D., Suetens, P.: Medical image registration using mutual information. In: Proceedings of the IEEE. Volume 91., IEEE (2003) 1699- 1722

[6] Hermosillo, G., Chefd'Hotel, C., Faugeras, O.: Variational methods for multimodal image matching. IJCV 50 (2002) 329-343

[7] D'Agostino, E., Modersitzki, J., Maes, F., Vandermeulen, D., Fischer, B., Suetens, P.: Free-form registration using mutual information and curvature regularization. In: WBIR. (2003) 11-20

[8] Gaens, T., Maes, F., Vandermeulen, D., Suetens, P.: Non-rigid multimodal image registration using mutual information. In: MICCAI. (1998) 1099-1106

[9] Leventon, M., Grimson, E.: Multi-modal volume registration using joint intensity distributions. In: MICCAI, Springer-Verlag (1998) 1057-1066

[10] Chung, A.C.S., Wells, W.M., Norbash, A., Grimson, W.E.L.: Multi-modal image registration by minimising Kullback-Leibler distance. In: MICCAI (2). (2002) $525-532$

[11] Gan, R., Wu, J., Chung, A.C.S., Yu, S.C.H., III, W.M.W.: Multiresolution image registration based on Kullback-Leibler distance. In: MICCAI (1). (2004) 599-606

[12] Zöllei, L., Fisher, J.W., III, W.M.W.: A unified statistical and information theoretic framework for multi-modal image registration. In: IPMI. (2003) 366-377

[13] Lorenzen, P., Davis, B., Gerig, G., Bullitt, E., Joshi, S.C.: Multi-class posterior atlas formation via unbiased Kullback-Leibler template estimation. In: MICCAI (1). (2004) 95-102

[14] Collins, D., Zijdenbos, A., Kollokian, V., Sled, J., Kabani, N., Holmes, C., Evans, A.: Design and construction of a realistic digital brain phantom. IEEE TMI 17 (1998) 463-468 